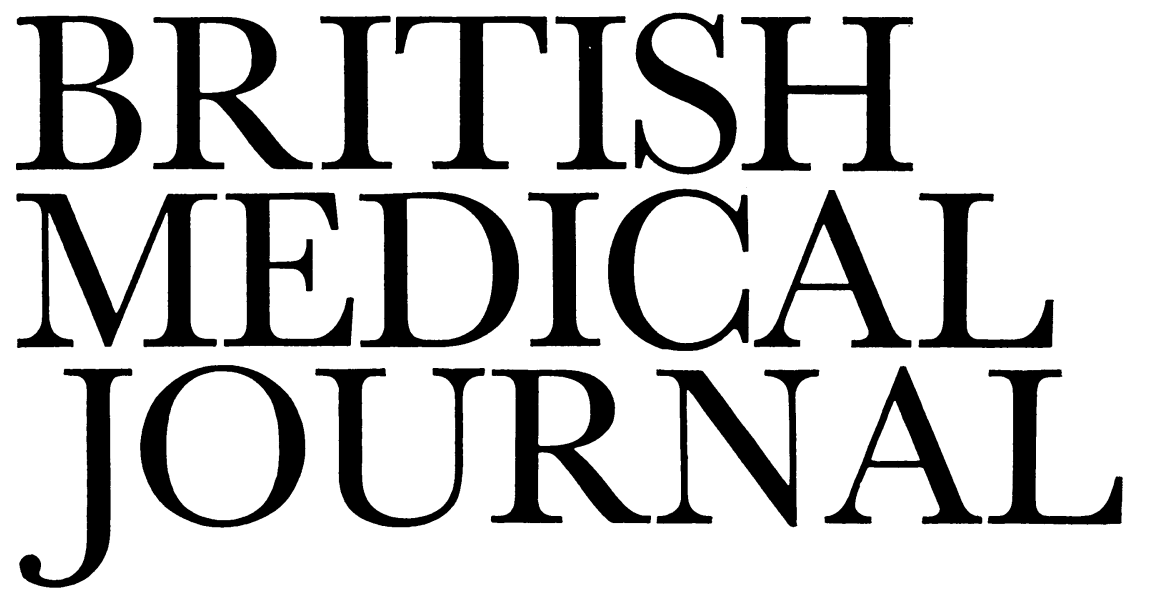

\title{
Sunscreens, photocarcinogenesis, melanogenesis, and psoralens
}

Most years in Britain, and regularly in sunnier countries, exposure to ultraviolet radiation takes its toll in terms of acute burns, photodermatoses, aging skin, and skin cancers. The high risk of skin cancers from exposure to the sun does not hit the headlines because almost all such lesions are completely curable. Nevertheless, the general public should be better informed about the harmful effects of actinic exposure as increasing leisure and a fashion for tanned skin are putting more and more people at risk. The pale Celtic skin tans poorly and is much more susceptible to damage than intrinsically darker skins, which tan more readily; melanin seems to be protective to a greater or less extent against all the adverse effects of ultraviolet radiation. So how far can skin protection be helped by provoking melanogenesis?

The most damaging part of the ultraviolet spectrum is in the waveband from 280 to $315 \mathrm{~nm}$-the UV-B range. The spectrum of terrestrial sunlight extends down to about $290 \mathrm{~nm}$, well into the UV-B and up into the UV-A range (315 to 400 $\mathrm{nm}$ ). Most suntan preparations include in their formulation substances which act as barriers to UV-B. People with normal skins generally use such preparations, if at all, only during their summer holidays, but patients with photodermatoses need to use sun barriers throughout much of the year. The "sun-protection factor" has been widely used in Europe and the United States, but rather less in Britain, as a measure of sunbarrier efficiency. The sun-protection factor is the ratio of the ultraviolet radiation exposure required to produce minimal sunburn erythema with the sunbarrier preparation in place to that required in the case of untreated skin. Sunbarrier preparations with a low sun-protection factor of, say, 2 would allow exposure to be doubled before sunburn occurred. These are suitable for people who tan readily and wish to avoid the more acute discomforts of sunburn. A sunbarrier preparation with a sun-protection factor as high as 15 should, in theory, afford protection all day long or at least for extended periods; these, which are the standby of preventive treatment for patients with abnormal photosensitivity, were recently accepted for NHS prescription as "borderline substances." The active ingredients, including cinnamates, aminobenzoates, salicylates, and benzimidazoles, act by absorbing ultraviolet radiation in the UV-B range. The more powerful sunbarrier preparations could, if widely used, largely prevent the epidemic of skin cancer that plagues the so-called "Celts of Australia." They also help in the control of xeroderma pigmentosum, and can prevent the acute symptoms in idiopathic photodermatoses such as polymorphous light eruption.

In the 1970s suntan preparations began to be marketed which contained not only chemicals with sunbarrier properties but also 5-methoxypsoralen, which promotes melanogenesis in the presence of UV-A. This substance is present in bergamot oil-widely used as a minor constituent in soaps, perfumes, and other cosmetic products. The mechanism whereby 5methoxypsoralen plus UV-A provokes melanogenesis is not clear but is possibly a non-specific response to injury. What is certain is that 5-methoxypsoralen can react with DNA to form photoadduct lesions in the DNA duplex; cell culture studies (bacterial, yeast, and mammalian) suggest that the effect is photomutagenic, and mice treated with 5-methoxypsoralen plus UV-A develop skin cancer. 5-Methoxypsoralen is one of a number of psoralens that have been used in conjunction with UV-A in the treatment of skin conditions such as vitiligo and psoriasis (PUVA therapy). Though the available information is largely anecdotal, there is no clear evidence that treatment with psoralens increases the risk of skin cancer in man, and there is apparently no excess risk of skin cancer in workers in the bergamot industry centred in Calabria. A follow-up study of patients with psoriasis treated with PUVA is still of relatively short duration, but Stern and his colleagues ${ }^{1}$ are finding a shift in the predominant kind of skin cancer in such patients from the basal cell to the squamous cell variety. Interpretation of the data is, however, complicated as many patients have histories of other potentially carcinogenic treatments-for example, ionising radiation.

A recent conference in Paris earlier this year on psoralens in cosmetics and dermatology provided a good opportunity for the benefits and risks of incorporating them in sunbarrier preparations to be discussed in a scientific quorum. The conference also provided a forum for an up-to-date review of the possible dangers of PUVA treatment. The photomutagenicity of both 8-methoxypsoralen and 5-methoxypsoralen in yeasts had already been reported, ${ }^{2}$ as had their photocarcinogenicity in mouse skin. ${ }^{3}$ The confirmation and extension of the latter result by Magnus and Young as reported in Paris suggested that it may not be prudent to incorporate psoralens into suntan preparations. Nevertheless, Lane-Brown from Sydney, Australia, reported enhanced tanning associated with the use of 
a sunbarrier preparation containing 5-methoxypsoralen and suggested that once psoralen had provoked a tan there might be improved protection from further damage from solar ultraviolet radiation. This might be especially important to those Australians whose skin tans poorly. At present, therefore, opinion is somewhat divided about the balance between risk and benefit for 5-methoxypsoralen as an ingredient of suntan preparations. In the case of PUVA treatment for severe psoriasis or vitiligo, however, the consensus view remains that the benefits far outweigh the risk of skin cancer.

Much research remains to be done on this subject. The mechanisms whereby psoralens cause photosensitivity, mutagenesis, and carcinogenesis are not clear. At the molecular level two types of photoadduct lesions with DNA have been described, one in which the psoralen is covalently bonded to only one DNA strand and another where the two strands of the duplex are cross-linked. Natural repair of psoralen-DNA lesions is probably much less well developed in rodents that in man. Hence the results of mouse studies with psoralens may be misleading. The cross-linking psoralen DNA lesion interferes with DNA synthesis-which may be how PUVA works in psoriasis-and this cross-linking DNA lesion has been thought to be more lethal, more mutagenic, and more carcinogenic than the single-strand lesion. Another view, however, is that because of its lethality the cross-linking DNA lesion is less likely to give rise to mutant cells or cancer. These conflicting views need to be resolved. Certain psoralens, such as angelicin, through steric hindrance form photoadducts binding only one DNA spiral, whereas other psoralens such as 8- and 5methoxypsoralen cause cross-links. If a "safe" psoralennatural or synthetic-could be identified it could replace 8methoxypsoralen in the PUVA treatment of psoriasis. And a "safe" psoralen which provoked tanning would be a highly desirable ingredient of sunbarrier preparations.

1 Stern RS, Thibodeau LA, Kleinerman RA, Parrish JA, Fitzpatrick TB. Risk of cutaneous carcinoma in patients treated with oral methoxsalen photochemotherapy for psoriasis. N Englf Med 1979;300:809-13.

2 Ashwood-Smith MJ, Poulton GA, Barker M, Mildenberger M. 5-Methoxypsoralen, an ingredient in several suntan preparations, has lethal, mutagenic and clastogenic properties. Nature $1980 ; 285: 407-9$.

${ }^{3}$ Zajdela F, Bisagni E. 5-Methoxypsoralen, the melanogenic additive in sun-tan preparations, is tumorigenic in mice exposed to $365 \mathrm{~nm} \mathrm{UV}$ radiation. Carcinogenesis $1981 ; 2: 121-7$.

\section{Drug-resistant tuberculosis}

Drug-resistant strains of Mycobacterium tuberculosis emerge whenever one of the antituberculosis drugs is given alone to heavily infected patients. Mutant bacilli are always present as a small proportion of the original bacterial population, and these overgrow sensitive organisms during treatment. This process was recognised very soon after streptomycin was first used in the treatment of tuberculosis ${ }^{1}$ and led to the policy of using one or more additional drugs to inhibit the growth of the naturally resistant strains. The efficiency with which a drug prevents resistance from emerging depends on whether it can inhibit the growth of all the bacilli in the lesions all of the time. Rifampicin and streptomycin are the most valuable drugs in preventing the emergence of drug resistance, while pyrazinamide is also useful because of its sterilising action. ${ }^{2}$

Attention to the importance of drug-resistant tuberculosis has recently been drawn by Aswapokee and colleagues ${ }^{3}$ from New York City and by the publication of a world atlas of initial drug resistance by the International Union Against Tuberculosis. ${ }^{4}$ The union's committee on bacteriology and immunology acknowledges a major problem with the reliability of its data because susceptibility testing of $M$ tuberculosis requires a high standard of laboratory skill, which varies from country to country. Factors affecting the comparison of reports of drug resistance in tuberculosis have been discussed by Horne. ${ }^{5}$ A difference has to be recognised between true primary resistance and undisclosed acquired resistance in patients who have concealed a history of previous chemotherapy. The two combined give a rate for initial drug resistance, and this varies widely from country to country. High rates, particularly to isoniazid, are recorded, for example, from Cairo and lower Egypt (34\%), India (26\%), Korea (24\%), and Thailand $(52 \%)$, and relatively low rates from Finland and Algeria (both $2 \%$ ). An average figure of $7 \%$ was recently recorded from 19 city and State laboratories in the United States, ${ }^{6}$ and one of $5 \%$ for Scotland. ${ }^{7}$ In a survey of 1038 isolates from patients in England and Wales in 1978-9 only $3 \%$ were resistant, $1.5 \%$ in whites and $7.5 \%$ in patients from the Indian subcontinent. ${ }^{8}$ Other studies have shown higher rates in immigrant populations. ${ }^{46}$ The overall rate is declining slowly in Britain.

The high rates of drug-resistant infections in some parts of the world are disquieting as a community problem. But what about the individual ? In patients infected with primary drugresistant organisms the disease responds well to chemotherapy provided that effective regimens are used-even in the absence of initial sensitivity testing. In a study in Hong Kong, where initial drug resistance is high, accurate tests of sensitivity before treatment gave surprisingly small benefits. Though $30 \%$ of patients in the study had initially drug-resistant organisms, quiescence was achieved in $89 \%$ of 187 patients when no notice was taken of the results of the sensitivity tests and in $92 \%$ when the regimen was adjusted to take account of the results of pretreatment tests. A quiescence rate of $95 \%$ was achieved in patients with fully sensitive organisms, though potentially the regimen used was $100 \%$ effective. ${ }^{9} 10$ In Britain only one out of every 200 patients with bacteriologically positive results who are given effective standard regimens would benefit from the routine use of accurate susceptibility tests. Such tests are, however, valuable in the intelligent management of patients with acquired resistance.

Short-course chemotherapy may be effective in the presence of initial drug resistance even to both isoniazid and streptomycin, provided that the regimen used includes streptomycin, isoniazid, rifampicin, and pyrazinamide for two months followed by rifampicin in the continuation phase. ${ }^{1112}$ When initial resistance to streptomycin, para-aminosalicylic acid, and isoniazid is frequent the drug regimen should contain both rifampicin and pyrazinamide, in part because of their special sterilising role and in part because initial resistance to them is infrequent. The level of resistance to rifampicin and pyrazinamide will, however, need to be watched with considerable interest and not a little anxiety in the next few years. If it is to remain low regimens will have to be used that largely overcome the influence of initial drug resistance. ${ }^{13} \mathrm{~A}$ disquieting survey of chemotherapy practices in 28 major metropolitan health departments in the United States has shown, however, that two-drug regimens (isoniazid with ethambutol or with rifampicin) were used most frequently in initial treatment; that only one-third of the programmes used three-drug regimens containing isoniazid, ethambutol, and rifampicin for initial treatment; and that only three of these used this regimen for more than $75 \%$ of their patients. ${ }^{14}$ 